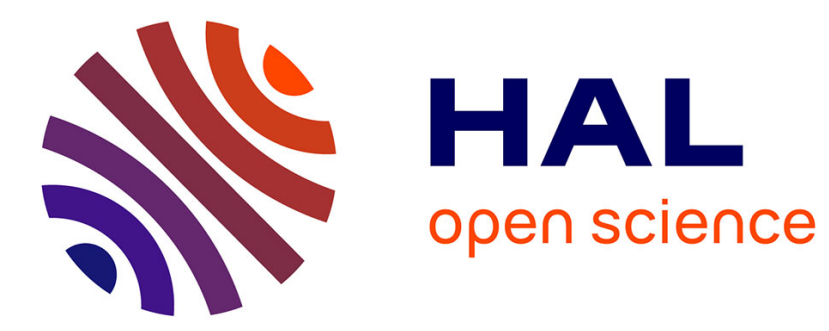

\title{
Polynomial Phase Signal Modeling Using Warping-Based Order Reduction
}

\author{
André Quinquis, Cornel Ioana, Emanuel Radoi
}

\section{To cite this version:}

André Quinquis, Cornel Ioana, Emanuel Radoi. Polynomial Phase Signal Modeling Using WarpingBased Order Reduction. International Conference on Acoustic, Speech and Signal Processing ICASSP 2004, May 2004, Montreal, Canada. pp.741-744, 10.1109/ICASSP.2004.1326364 . hal-00317938

\section{HAL Id: hal-00317938 \\ https://hal.science/hal-00317938}

Submitted on 3 Sep 2008

HAL is a multi-disciplinary open access archive for the deposit and dissemination of scientific research documents, whether they are published or not. The documents may come from teaching and research institutions in France or abroad, or from public or private research centers.
L'archive ouverte pluridisciplinaire HAL, est destinée au dépôt et à la diffusion de documents scientifiques de niveau recherche, publiés ou non, émanant des établissements d'enseignement et de recherche français ou étrangers, des laboratoires publics ou privés. 


\title{
POLYNOMIAL PHASE SIGNAL MODELING USING WARPING-BASED ORDER REDUCTION
}

\author{
André Quinquis, Cornel Ioana, Emanuel Radoi \\ ENSIETA, 2 rue François Verny, Brest - FRANCE \\ E-mail : ioanaco@ensieta.fr, quinquis@ensieta.fr
}

\begin{abstract}
The high-order ambiguity function (HAF) was introduced for the estimation of polynomial-phase signals (PPS). Currently the HAF suffers from noise-masking effects and from the appearance of undesired cross terms in the presence of multi-components PPS. The multi-lag product HAF concept was then proposed as a way to improve the performances of the HAF. Nevertheless, performances of the new methods are affected by the error propagation. This effect is due to the technique used for polynomial order reduction, common for current approaches : signal multiplication with the complex exponentials formed with the estimated coefficients.

In this paper, we introduce an alternative method to reduce the polynomial order, based on the successive unitary signal transformation, according to each polynomial order. We will prove that this method considerably reduces the effect of error propagation.
\end{abstract}

\section{INTRODUCTION}

It is well known that there is no transformation from the Cohen's class which can produce the complete concentration along the instantaneous frequency law (IFL) when this one is a nonlinear function of time. Therefore, different high order distributions have been developed in order to better match the non-linear timefrequency behavior of the analyzed signal [1], [2], [3], [4]. For example, the polynomial phase signal constitutes a good model in a variety of applications (e.g. Radar, Communication, etc ) [1].

One of the first approaches to estimate the parameters of the PPSs [2] provides good results for high signal-tonoise ratio (SNR). Nevertheless, since the HAF is a nonlinear method, it suffers from three basic problems : 1) noise-masking effects for low SNR, 2) cross terms in the presence of multi-component PPSs (mc-PPSs) and 3) the propagation of the approximation error from an order to other.

Recently, different methods have been proposed in order to eliminate the first two limitations. The key point is to use the multi-lag concept in the HAF computing procedure [1]. Moreover, multiplying the HAFs obtained for some lag sets (product HAF - PHAF), the performances related to 1) and 2) are considerably improved with respect to multi-lags HAF [1].

Nevertheless, the effect of propagation error remains a serious limitation when we try to estimate a deeply nonlinear IFL (underwater transitory signals, digital modulation, etc). Therefore, we propose a new procedure for polynomial order compensation, based on the recursive signal warping. In fact, using the order reduction property of the warping technique, the idea is to iteratively apply this method to reduce the phase orders.

This paper is organized as follows. In section 2, we present the Product HAF method. The major limitation of the PHAF, related to the error propagation effect, is described in section 3. Afterwards, using the warping technique [5], a new method for order compensation is proposed in section 4. Some examples will be presented in section 5. We will finally present some remarks in "Conclusion and Perspective" (section 6).

\section{PRODUCT HIGH-ORDER AMBIGUITY FUNCTION}

As it was illustrated in [1], [2], the classical HAF algorithm presents some limitations, related to the noise robustness and the cross-terms presence. In order to solve these aspects, the multi-lag HAF (mlHAF) concept has been initially proposed in [1]. In fact, the mlHAF is based on the generalization of the high order instantaneous moment HIM [2] :

$$
H M_{K}\left[s(t) ; \boldsymbol{\tau}_{K-1}\right]=H M_{K-1}\left[s\left(t+\tau_{K-1}\right) ; \boldsymbol{\tau}_{K-2}\right] H M_{K-1}^{*}\left[s\left(t-\tau_{K-1}\right) ; \boldsymbol{\tau}_{K-2}\right]
$$

where $\boldsymbol{\tau}_{\mathbf{i}}=\left(\tau_{1}, \tau_{2}, \ldots, \tau_{i}\right)$ is the lag set. Applying the Fourier transform to (1), we obtain the ml-HAF of the signal $s(t)$ :

$$
m l H A F_{K}[s ; \alpha, \boldsymbol{\tau}]=\int_{-\infty}^{\infty} H I M_{K}[s(t) ; \boldsymbol{\tau}] e^{-j \alpha t} d t
$$

The main property of HIM is that, assuming a PPS model for the analyzed signal, i.e.

$$
s(t)=A \exp j \phi(t)=A \exp \left[j \sum_{k=0}^{K} a_{k} t^{k}\right]
$$

the $K^{\text {th }}$ order HIM is reduced to a harmonic with amplitude $A^{2^{k-2}}$, frequency $\widetilde{\omega}$ and phase $\widetilde{\phi}$ : 


$$
H I M_{k}[s(t) ; \boldsymbol{\tau}]=A^{2^{k-1}} \exp j\left(\tilde{\omega}_{k} \cdot t+\tilde{\phi}_{k}\right)
$$

where $\widetilde{\omega}_{k}=k ! \tau^{K-1} a_{k}(5)$.

Based on these results, Porat [2] has proposed an algorithm which estimates sequentially the coefficients $\left\{a_{k}\right\}$. At each step, using a spectral analysis method, we estimate the spectral peak and, using the HAF, we compute an estimation value $\left(\hat{a}_{k}\right)$ of $a_{\mathrm{k}}$. Using this value, the effect of the phase term of the higher order is removed:

$$
s^{(k-1)}(t)=s^{(k)}(t) \cdot e^{-j \hat{a}_{k} t^{k}}
$$

Using the ml-HIM concept (relation (1)), Barbarossa and al [1] introduced the Product HAF: the mlHAFs computed, via relation (2), for different lag sets

$$
\mathbf{T}=\left\{\begin{array}{c}
(l) \\
\boldsymbol{\tau}_{K-1}^{(l)}
\end{array}\right\}_{l=\overline{l, L}} ; \boldsymbol{\tau}_{K-1}^{(l)}=\left\{\tau_{i}\right\}_{i=\overline{1, K-1}}
$$

are multiplied, obtaining also a more robust method and a cross-term free representation :

$$
\operatorname{PHAF}(f ; \mathbf{T})=\prod_{l=1}^{L} m l H A F_{K}\left[s ; \frac{\prod_{i=1}^{K-1} \tau_{i}^{(l)}}{\prod_{i=1}^{K-1} \tau_{i}^{(1)}} f, \boldsymbol{\tau}_{K-1}^{(l)}\right]
$$

The simulation results given in [1] proved that the PHAF solves both noise robustness and ambiguity problems, providing also a correct IFL estimation. Nevertheless, the analyzed signals were characterized by smooth time-frequency behavior (usually, $3^{\text {th }}$ order PPs). If this condition is not verified, one of the major limitation of the PHAF based approaches, related to the error propagation phenomenon, acts. This phenomenon is studied in the next section.

\section{ERROR PROPAGATION IN POLYNOMIAL PHASE MODELING}

Let consider the signal given in (3) and we denote with $\hat{a}_{K}$ the estimation of the $k^{\text {th }}$ order polynomial coefficient. In real applications [2], since a spectral estimation of a discrete sequence is involved, this value differs from the theoretic one by $\varepsilon_{K}=a_{K}-\hat{a}_{K}$ - the approximation error. This error is directly related to the number of points in Fourier transform and to the SNR ([1]). Using this estimate, we remove, via (6), the $k$ order phase component

$$
s^{(K-1)}(t)=A e^{j\left(\sum_{k=0}^{K-1} a_{k} t^{k}+\left(a_{K}-\hat{a}_{K}\right) t^{K}\right)}=A e^{j \sum_{k=0}^{K-1} a_{k} t^{k}} \cdot e^{j \varepsilon_{K} t^{K}}
$$

The $(k-1)^{\text {th }}$ order HIM of $s^{(k)}(t)$ is expressed as

$$
H I M_{K-1}\left[s^{(K-1)}(t) ; \tau\right]=e^{j\left[(K-1) ! \tau^{K-2} \hat{a}_{K-1} t+\hat{\phi}_{K-1}\right]}
$$

This expression is a consequence of the main HAF property previously presented : the $(k-1)^{\text {th }}$ order HIM of a PPS is a sinusoid with an angular frequency related, via $(5)$, to the $(k-1)^{\text {th }}$ order polynomial coefficient. Nevertheless, due to the measurement error existing for the $k^{\text {th }}$ order, $\hat{a}_{K-1}$ is not the correct value of the $(k-1)^{\text {th }}$ order polynomial coefficient of signal $s^{(k-1)}$. In order to find the relation between the errors at order $k$ and $(k-1)$, we can evaluate $H I M_{K-1}\left[s^{(K-1)} ; \tau\right]$ using the recurrent definition given in (1) and the relation (4) and (5). We obtain the following expression :

$$
\operatorname{HIM}_{K-1}\left[S^{(K-1)} ; \tau\right]=e^{j\left[(K-1) !\left(\tau^{K-2} a_{K-1}+K \varepsilon_{K} \tau^{K-1}\right) t+R\right]}
$$

where $R$ is a residual term which does not depend on $t$. Since the mlHAF evaluation supposes the computation of Fourier transform of HIM, we focus only on the coefficient of $t$. Therefore, if we compare the corresponding terms which appear in (10) and (11) we obtain the following relation between the errors for two consecutive orders :

$$
a_{K-1} \tau^{K-2}+K \varepsilon_{K} \tau^{K-1}=\hat{a}_{K-1} \tau^{K-2}
$$

and, with the notation $\varepsilon_{K-1}=\hat{a}_{K-1}-a_{K-1}$, we obtain :

$$
\varepsilon_{K-1}=K \varepsilon_{K} \tau
$$

Furthermore, using the optimal value of the lag proposed in [2] i.e. $\tau=N / K(N$-sample number $)$, we get:

$$
\varepsilon_{K-1}=N \varepsilon_{K}
$$

This relation shows that the error existing at a given order is transmitted at the inferior order by multiplication of $N$. The next figure illustrates this dependence for $N=10$.

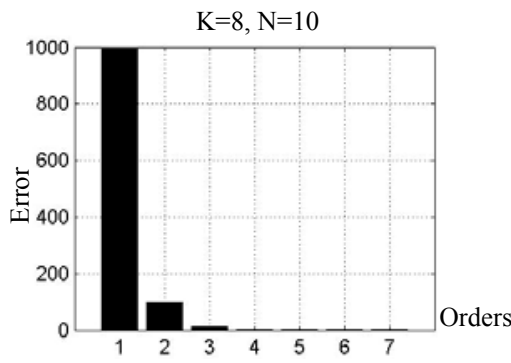

Figure 1. Error propagation effect.

From this figure, it can be observed that even if the measurement error for the highest order is insignificant, its effect through the lower orders becomes deeply disturbing. It explains why the error propagation effect does not affect the polynomial estimation when a small approximation order is required (3 or 4). Nevertheless, there are many situations which impose a high 
approximation order : digital modulations, transitory signals, etc. One example is given in figure 2 where we process, via PHAF-based phase modeling method, a frequency shift keying (FSK) modulation.
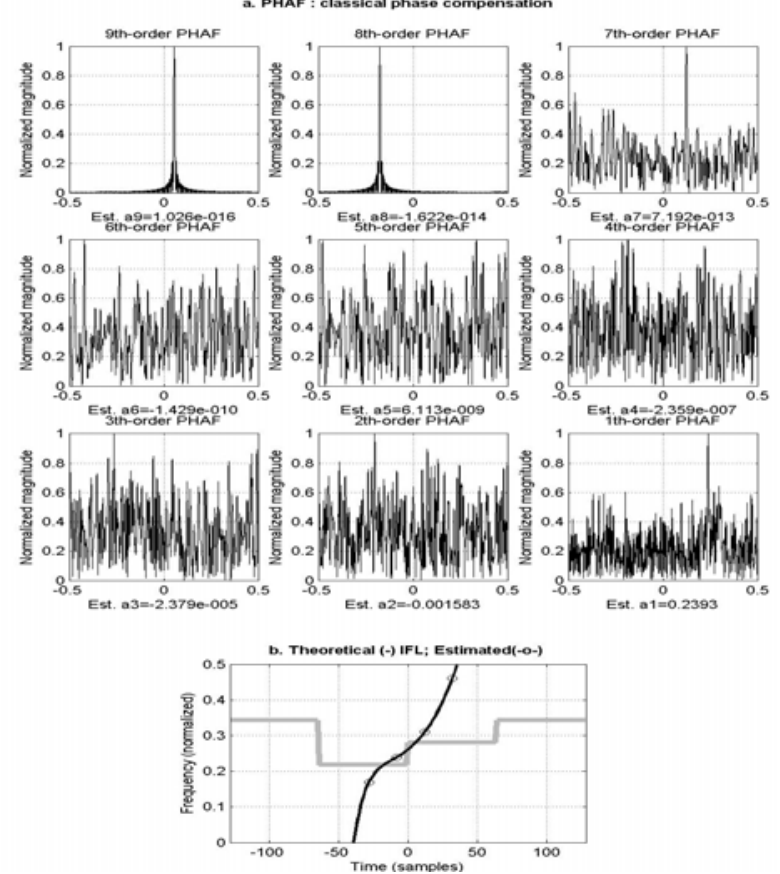

Figure 2. PHAF-based phase modeling of a FSK signal

The theoretical IFL is plotted in figure 2.b. Note that the SNR is about $30 \mathrm{~dB}$. The PHAF-based estimation procedure is applied, starting with the order 9. At the superior orders $(9,8,7)$ PHAF performs quite well : the propagation error is insignificant, but its effect is accumulated and it becomes disturbing for inferior orders (down to 6). This can be observed in the figure 2.a. , where the peak locations give information about the estimation quality. Consequently, the estimation of the polynomial coefficients is not correct (figure 2.b); the evaluated IFL does not match the correct time-frequency behavior of the FSK modulation.

A potential solution to reduce the error propagation is proposed in next section.

\section{WARPING-BASED PHASE ORDER REDUCTION}

In time-frequency analysis, the warping operator principle has recently become a very useful concept for generation of non-linear time-frequency representation (TFR) [5]. Mathematically, a warping operator is defined as a unitary transform $\mathbf{U}$ on $l^{2}(\mathfrak{R})$, whose effect is $x$-axis deforming :

$$
(\mathbf{U} s)(x)=\left|w^{\prime}(x)\right|^{1 / 2} s[w(x)]
$$

where $w$ is a smooth, one-to-one function, comprising a large subclass of unitary transformations ([5]). Generally, these functions, called warping functions, are chosen to ensure the "linearization" of signal time-frequency behavior. The design of a warping function associated to a signal is possible if the signal modulation nature is known.[5]. This is not always possible, especially in the case of passive systems. For this case, the polynomial phase modeling seems to be a potential solution for signal IFL describing.

To reduce the error propagation effect, we propose the replacement of this technique with a warping-based method. More precisely, we define a particular warping operator as :

$$
\mathbf{w}_{\mathbf{K}}: t \stackrel{\mathbf{w}_{\mathbf{K}}}{\longrightarrow} t_{w}^{(K)}=\mathbf{w}_{\mathbf{K}}(t)=\left(\frac{t}{\left|\hat{a}_{K}\right|}\right)^{1 / K}
$$

where $\hat{a}_{k}$ is the $k^{\text {th }}$ order estimated polynomial coefficient. The following example illustrates the effect of this warping operator for a $3^{\text {th }}$ order PPS given by :

$$
s(t)=e^{j 2 \pi\left(0.25 t-4.6 \cdot 10^{-4} \cdot t^{2}+3 \cdot 10^{-6} \cdot t^{3}\right)}
$$

The Wigner-Ville Distribution (WVD) of this signal is depicted in figure 3 . Using the warping operator obtained by the particularization of the relation (16) (for $\mathrm{k}=3$ and $\left.\hat{a}_{3}=2.8 \cdot 10^{-6}\right)$ we obtain a linear time-frequency structure (i.e. a $2^{\text {th }}$ order PPS) as illustrated in figure 3.
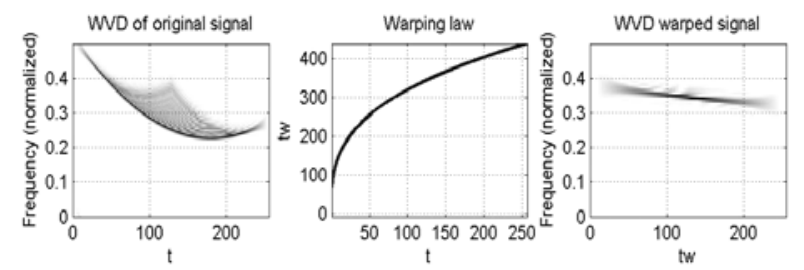

Figure 3. Order reduction using the warping operator (16)

Therefore, in order to reduce the polynomial order we apply, in an iterative manner, a warping operator designed as shown in (16). The effect of this operator on the PPS defined in (3) is depicted by :

$$
\begin{gathered}
\left(\mathbf{U}_{\mathbf{K}} y\right)\left(t_{w}^{(K)}\right)=A \exp \left\{j a_{K}\left[\left(\frac{t}{\left|\hat{a}_{K}\right|}\right)^{1 / K}\right]^{K}\right\} \cdot \exp \left\{j \sum_{m=0}^{K-1} a_{m}\left[t_{w}^{(K)}\right]^{m}\right\} \\
=A \underbrace{A \exp \left\{j \sum_{m=0}^{K-1} a_{m}\left[t_{w}^{(K)}\right]^{m}\right\}}_{(K-1)^{1 n} \text { order PPS }} \underbrace{\exp \left\{j \frac{a_{K}}{\hat{a}_{K} \mid} t\right\}}_{\text {residual }}
\end{gathered}
$$

Therefore, the result of the warping transform of a $K^{\text {th }}$ order PPS consist in a $(K-1)^{\text {th }}$ order PPS for new temporal variable $t_{w}^{(K)}$. The $(K-1)^{\text {th }}$ order PHAF of this signal, with respect to variable $t_{w}^{(K)}$, peaks to a frequency location related, via relation (5), to the $a_{\mathrm{K}-1}$ coefficient. Once $a_{\mathrm{K}-1}$ estimated, we construct the $(K-1)^{\text {th }}$ order unitary operator $\mathbf{U}_{\mathbf{K}-1}$ as indicated in (16). The process is iterated until all polynomial coefficients are estimated. 
As the relation (18) shows, the $(\mathrm{K}-1)^{\mathrm{th}}$ coefficient of the signal $\left(\mathbf{U}_{\mathrm{K}} y\right)$ depends only on the $a_{\mathrm{K}-1}$ coefficient, avoiding also the process of error propagation to the lower orders. This will be practically proved in the next section.

\section{SIMULATION RESULTS}

In this section, we firstly give the performances in terms of estimated variances as a function of the SNR. We assumed a $3^{\text {th }}$ order PPS embedded in white gaussian noise. Two methods were compared - classical PHAF estimation method and PHAF-based estimation method with warping-based phase compensation (denoted "WarpComp" method). Each variance was computed for 500 trials and, for each order, it was compared with the Cramer-Rao bound (CRB), theoretically evaluated in [2].
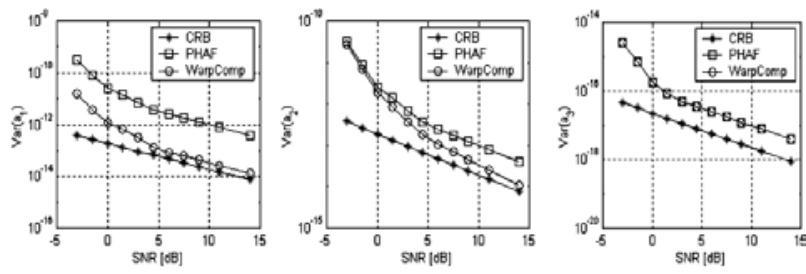

Figure 4. The estimated variances versus SNR

The first plot proves that, for the highest order, the performances of both methods are similar : the estimation of the highest order coefficient depends on the noise only. The next two pictures show that, using the warping-based phase compensation, the estimation performances remains close to the CRB as in the case of the highest-order coefficient. Consequently, the performances of this method depend only on the noise, whereas in the PHAF case they are affected also by the error propagation phenomenon.

The error propagation effect reduction is also illustrated in the next figure, using the signal presented in the figure 2. This figure shows, the proposed method provides a much more accurate estimation of the IFL of a FSK signal. It is illustrated by an existence of a single peak at each polynomial order. Consequently, the polynomial order reduction through the procedure based on the warping operators (section 4) considerably improves the performances of the PHAF-based estimation procedure.

The statistical analysis and the result for a FSK signal prove that this method can successfully be used to estimate the polynomial model of a general class of signals.
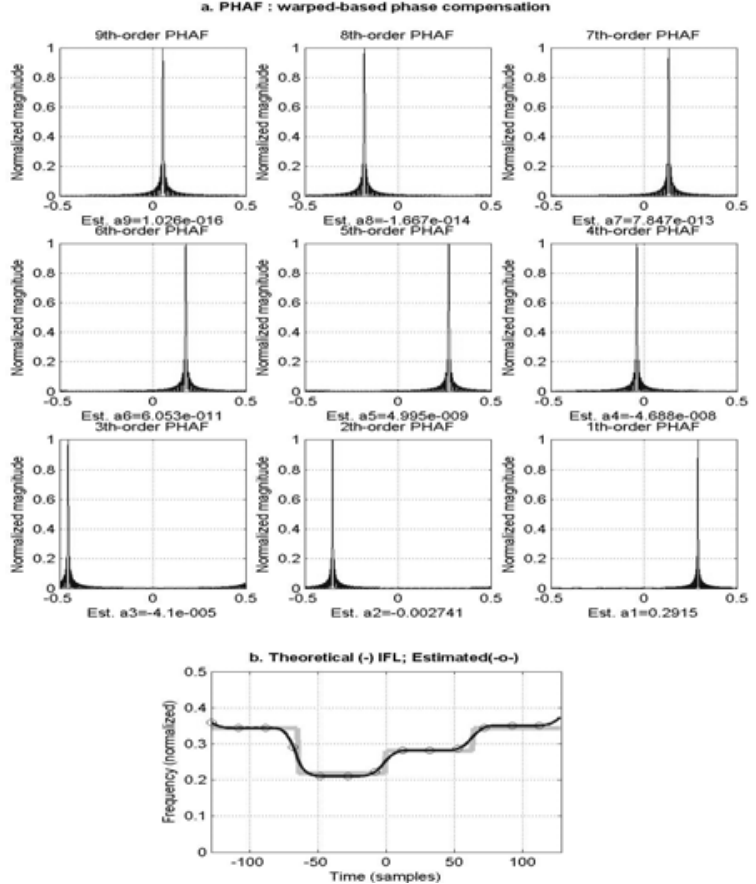

Figure 5. PHAF estimation using warping phase reducing

\section{CONCLUSION AND PERSPECTIVES}

In this paper we have proposed a method, for polynomial order reduction, based on the warping principle, applied to each polynomial order. This method constitutes an attractive way to attenuate the effect of error propagation which inherently appears in any estimation algorithm of the PPSs. As shown by the results, this method, associated with a modern procedure for polynomial phase modeling, provides accurate timefrequency information about the analyzed signal.

In further works, we intend to apply this procedure in real contexts, such underwater signal processing or digital modulation recognition.

\section{REFERENCES}

[1] S. Barbarossa, A. Scaglione, G.B. Giannakis, "Product HighOrder Ambiguity Function for Multicomonent Polynomial-Phase Signal Modeling”, IEEE Trans. On Signal Processing, vol. 46, No. 3, March 1998.

[2] B. Porat, Digital Processing of Random Signals, Pretince Hall, New Jersey, 1993.

[3] B. Boashash, P.O'Shea, "Polynomial Wigner-Ville distributions and their relationship to time-varying higher order spectra", IEEE Trans. On Signal Processing, vol.42, pp. 216220, Jan. 1994.

[4] L.J. Stankovic, "L-class of time-frequency distributions", IEEE Signal Processing Lett., vol.3, pp. 22-25, Jan. 1996.

[5] R. Baraniuk, "Unitary Equivalence : a new twist on signal processing”, IEEE Trans. On Signal Processing, vol. 43, no. 10, Oct. 1995. 